\title{
ÍNDICE DE ASTRÓCITOS GEMISTOCÍTICOS E IMUNO-EXPRESSÃO DA PROTEÍNA P53 EM ASTROCITOMAS, GRAU II E III OMS
}

\author{
Dely Cristina Martins ${ }^{1}$, João Norberto Stávale ${ }^{1}$, \\ Suzana Maria Fleury Malheiros ${ }^{2}$, Lucila Heloisa Simardi Santiago ${ }^{1}$, \\ Leonor Cristina Manoja Roman ${ }^{1}$, Kátia Cilene Carozzi Aguiar ${ }^{1}$
}

\begin{abstract}
RESUMO - Foram estudados, retrospectivamente, 22 pacientes com diagnóstico de astrocitomas grau II ( $n=17)$ elll ( $n=5)$, OMS, no período de 1990 a 1998, cujos laudos histopatológicos descreviam a presença gemistocitos com o objetivo de determinar o índice de astrócitos gemistocíticos, investigar a imuno-expressão da proteína p53 e confrontá-los com o intervalo até a recorrência da neoplasia. O índice de astrócitos gemistocíticos, em cada caso, foi calculado a partir da razão entre o número de gemistocitos e o número total de células neoplásicas contadas, no mínimo 1000. Imuno-expressão nuclear da proteína p53 foi avaliada em astrócitos e gemistocitos neoplásicos; tanto a freqüência (7/22), como o índice de imuno-expressão positiva da p53 em gemistocitos, independentemente do grau histológico da neoplasia, foram inferiores aos relatados na literatura. Não se observou correlação entre o índice de astrócitos gemistocíticos e a imuno-expressão positiva da p53.
\end{abstract}

PALAVRAS-CHAVE: gemistocito, p53, astrocitoma, glioma.

\begin{abstract}
Fraction of gemistocytic astrocytes and immunoexpression of p53 protein in astrocytomas grade II and III (WHO)

ABSCTRACT - Twenty-two patients with astrocytomas, grade II or III WHO, were studied from 1990 to 1998. In all cases, histopathology showed that the astrocytomas had a gemistocytic component. The aims of this study were to establish the fraction of gemistocytic astrocytes, to investigate p53 protein immunoexpression and to evaluate correlations between these two parameters with the tumour outcome. Tumor cells were quantified at high-power magnification (x400). At least 1000 neoplastic cells (small neoplastic astrocytes plus gemistocytes) were counted in each specimen. The percentage of gemistocytes was defined as the gemistocytic index. Nuclear expression of p53 protein was evaluated in neoplastic astrocytes and gemistocytes. Both the frequency (7/22) as well the p53 immunoexpression indices in gemistocytes, regardless of the grade of the astrocytomas, were inferior from those reported in the literature. No correlation was found between the gemistocytic indices and the p53 immunoexpression.
\end{abstract}

KEY WORDS: gemistocytes, p53, astrocytoma, glioma.

Astrocitomas difusos abrangem grande espectro de neoplasias desde lesões que crescem lentamente, até tumores altamente agressivos e fatais. Uma característica importante desse grupo de neoplasias éa tendência à recorrência, frequentementeacompanhada da progressão para fenótipos mais malignos, o que é um aspecto particular dos astrocitomas gemistocíticos. Gemistocitos não são observados no tecido cerebral normal ou durante a embriogênese ${ }^{1-3}$. Entretanto, sua presença em neoplasias tem sido considerada um fator de pior prognóstico ${ }^{4}$. Vários estudos têm mostrado que a evolução dos astrocitomas para graus maiores de anaplasia, reflete aquisição seqüencial de alterações genéticas ${ }^{5-7}$. Como nos astrocitomas de baixo grau, mutações no gene p53 são as alterações genéticas mais comuns e estão presentesem freqüênciassemelhantesnosastrocitomas anaplásicose glioblastomas, sugere-se quesejam evento inicial no processo de transformação maligna ${ }^{6,8-10}$.

Os objetivos do presente estudo foram determinar o IAG, investigar a imuno-expressão da proteína p53 e correlacioná-los com o intervalo até a recorrência em astrocitomas, grau II e III OMS (Organização Mundial da Saúde).

\section{MÉTODO}

Foram estudados, retrospectivamente, 22 pacientes com diagnóstico de astrocitomas grau II $(n=17)$ e III $(n=5)$, OMS, no período de 1990 à 1998, cujos laudos histopatológicos descreviam a presença de astrócitos gemistocíticos.

Universidade Federal de São Paulo (UNIFESP-EPM), São Paulo SP, Brasil: ${ }^{1}$ Departamento de Patologia; ${ }^{2}$ Disciplina de Neurologia.

Recebido 9 Março 2001, recebido na forma final 10 Julho 2001. Aceito 21 Julho 2001.

Dr. João Norberto Stávale - Rua Botucatu 740 - 04023-900 São Paulo SP - Brasil. E-mail: stavale@ originet.com.br 
O IAG, em cada caso, foi estabelecido a partir da razão entre o número de gemistocitos e o número total de células neoplásicas contadas (astrócitos neoplásicos e astrócitos gemistocíticos), no mínimo $1000^{11,12}$. Para investigação da proteína p53 foi utilizado o método imuno-histoquímico com o anticorpo monoclonal D0-7, DAKO, na diluição 1:50. Considerou-se, como imuno-expressão positiva para a p53, qualquer percentagem de núcleos corados. O intervalo até a recorrência da neoplasia foi determinado a partir do dia da cirurgia, até a data da manifestação clínica e/ou do exame de imagem correspondente à recorrência.

Foi utilizado o teste de Kolmogorov-Smirnov para avaliar a distribuição dos valores obtidos para os índices e a normalidade destas observações. Para estudar a relação entre os índices, foram calculados coeficientes de correlação de Spearman. Essa relação também foi avaliada, classificando os pacientes em dois grupos (come sem astrócitos positivos para a p53) e comparando-se os grupos em relação ao IAG, sendo utilizado nesta comparação o teste t de Student.

A recorrência foi avaliada através do método de Kaplan Meier. Para estudar a relação entre a recorrência do tumor e o IAG, bem como com a imuno-expressão positiva da proteína p53 foi utilizado o teste de Log Rank. O nível de significância adotado foi de $5 \%$. Os processamentos foram realizados através do programa SPSS para Windows.

\section{RESULTADOS}

O índice médio de astrócitos gemistocíticos foi $17,53 \%$ (erro padrão $=1,3$ ), sendo o menor valor encontrado de $8,07 \%$ e o maior $26,70 \%$. A imunoexpressão nuclear da proteína p53 foi observada em ambas as células neoplásicas, astrócitos e gemistocitos (Fig 1), em 12 casos (54,54\%). Observou-se índice médio de astrócitos com expressão positiva de $4,57 \%$, com alta variabilidade (de 0 até $17,20 \%$ ). A proporção média de astrócitos gemistocíticos com imuno-expressão da $\mathrm{p} 53$ foi 0,24 . Dos 12 pacientes que tinham astrócitos positivos para p53, em 5, nenhum era gemistocítico, totalizando 15 pacientes com valores nulos e, portanto, apenas 7 (31,81\%) pacientes com imuno-expressão da p53 em gemistocitos (Tabela 2). O teste de Kolmogorov Smirnov utilizado mostrou hipótese de distribuição normal rejeitada, tanto para o IAG, como para o de astrócitos com imuno-expressão positiva da p53 e para o de astrócitos gemistocíticos com imuno-reatividade para a p53. Assim, como estes índices não eram normalmente distribuídos, para estudar a associação entre eles, foram calculados os coeficientes de correlação de Spearman. Os coeficientes foram praticamente nulos, com altos índices positivos da p53 sendo encontrados tanto para pacientes com índice de astrócitos gemistocíticos baixo, quanto para alto.

Dos 22 pacientes acompanhados, 11 tiveram recidiva do tumor, sendo que destes 2 foram a óbito. A curva de Kaplan Meier para avaliar o tempo de reaparecimento do tumor indicou que aos 3 anos após a cirurgia, a probabilidade livre de recidiva é

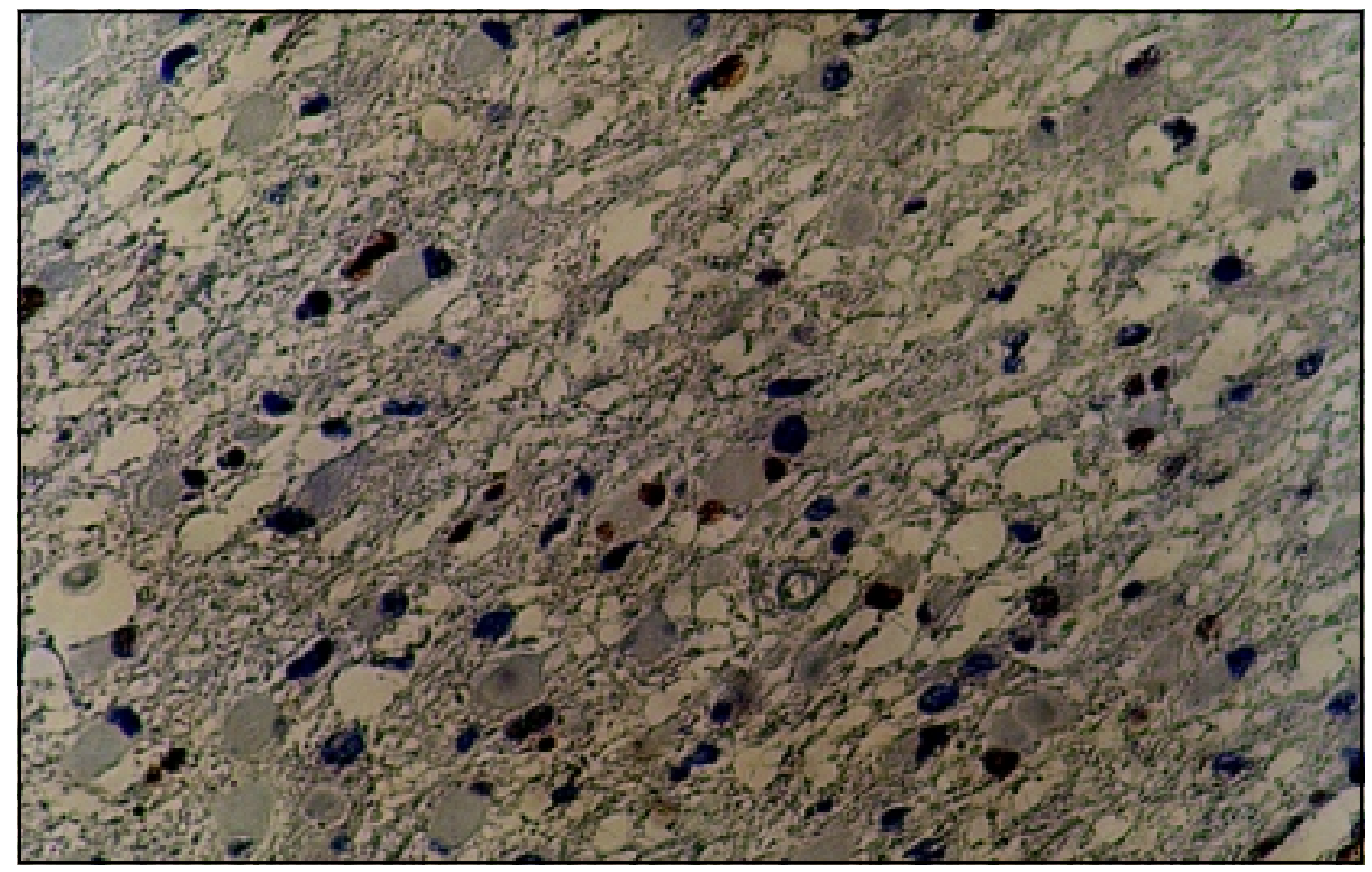

Fig 1. Fotomicrografia de astrocitoma grau II (OMS), com expressão imunohistoquimica nuclear da proteina p53 em gemistocitos (IH $400 \mathrm{x})$. 
Tabela 1. Relação dos pacientes segundo sexo, idade, grau histológico do tumor, IAG, IAp53+, IAGp53+ e seguimento.

\begin{tabular}{|c|c|c|c|c|c|c|c|c|c|c|}
\hline Paciente & Sexo & Idade & $\begin{array}{l}\text { Grau } \\
\text { hist }\end{array}$ & IAG & $\begin{array}{c}\text { IA } \\
\mathrm{p} 53+\end{array}$ & $\begin{array}{c}\text { IAG } \\
\text { p53+ }\end{array}$ & $\begin{array}{l}\text { Data da } \\
\text { cirurgia }\end{array}$ & $\begin{array}{l}\text { Data da } \\
\text { recorrência }\end{array}$ & $\begin{array}{c}\text { Data } \\
\text { do óbito }\end{array}$ & $\begin{array}{c}\text { Data } \\
\text { ult aval }\end{array}$ \\
\hline 1 & $M$ & 36 & II & 26,70 & 15,32 & 2,75 & $16 / 03 / 90$ & out/91 & - & $02 / 10 / 91$ \\
\hline 2 & $M$ & 25 & II & 17,18 & 8,99 & 0,33 & $23 / 11 / 90$ & jun/93 & 15/10/93 & - \\
\hline 3 & M & 49 & II & 24,00 & 0 & 0 & $21 / 03 / 91$ & mar/95 & - & $\mathrm{abr} / 00$ \\
\hline 4 & $M$ & 33 & II & 24,51 & 17,20 & 1,29 & 09/07/91 & - & - & $10 / 12 / 91$ \\
\hline 5 & $M$ & 49 & II & 8,17 & 6,99 & 0 & $10 / 10 / 91$ & set/98 & - & $18 / 11 / 98$ \\
\hline 6 & $M$ & 18 & II & 24,68 & 0 & 0 & $06 / 11 / 91$ & - & - & $01 / 04 / 92$ \\
\hline 7 & $\mathrm{~F}$ & 42 & III & 13,97 & 5,02 & 0,29 & $18 / 05 / 92$ & jan/00 & - & $31 / 01 / 00$ \\
\hline 8 & $M$ & 38 & II & 12,97 & 12,73 & 0 & $06 / 01 / 93$ & - & - & $14 / 06 / 94$ \\
\hline 9 & $M$ & 27 & II & 16,28 & 0 & 0 & $21 / 01 / 93$ & - & - & nov/94 \\
\hline 10 & $M$ & 22 & II & 8,07 & 5,18 & 0,20 & $28 / 04 / 93$ & nov/96 & - & jun/00 \\
\hline 11 & M & 41 & III & 10,97 & 9,57 & 0,30 & $27 / 12 / 94$ & dez/98 & - & jun/00 \\
\hline $12^{*}$ & $\mathrm{~F}$ & 52 & II & 13,55 & 0 & 0 & $23 / 05 / 95$ & - & 01/09/96 & - \\
\hline 13 & M & 07 & II & 11,76 & 0 & 0 & $03 / 05 / 96$ & - & - & $07 / 05 / 96$ \\
\hline 14 & $F$ & 30 & II & 21,82 & 0 & 0 & $13 / 01 / 98$ & dez/99 & - & $06 / 12 / 99$ \\
\hline 15 & M & 36 & II & 16,83 & 4,73 & 0 & $05 / 02 / 98$ & - & - & $\mathrm{NI}$ \\
\hline 16 & $\mathrm{~F}$ & 34 & III & 21,89 & 0 & 0 & $02 / 04 / 98$ & $\mathrm{fev} / 00$ & - & $26 / 06 / 00$ \\
\hline 17 & $M$ & 33 & III & 16,45 & 0 & 0 & $23 / 04 / 98$ & - & - & $05 / 06 / 00$ \\
\hline 18 & $M$ & 36 & III & 23,69 & 4,17 & 0 & $17 / 08 / 9 /$ & - & - & jun/00 \\
\hline 19 & $M$ & 37 & II & 20,04 & 5,46 & 0,10 & jan/96 & - & - & out/98 \\
\hline 20 & $\mathrm{~F}$ & 26 & II & 12,21 & 0 & 0 & $20 / 03 / 96$ & abr/98 & mar/99 & - \\
\hline 21 & $\mathrm{~F}$ & 27 & II & 26,20 & 0 & 0 & jul/97 & - & - & nov/99 \\
\hline 22 & $\mathrm{~F}$ & 28 & II & 13,83 & 5,19 & 0 & nov/98 & mai/99 & - & mar/00 \\
\hline
\end{tabular}

Grau hist, grau histológico do tumor; IAG, índice de astrócitos gemistocíticos; IAp53+, índice de astrócitos com imuno-expressão positiva da p53; IAGp53+, índice de astrócitos gemistocíticos com imuno-expressão positiva da p53; NI, não informado; Data ult aval: data da última avaliação; *paciente com diagnóstico através de biópsia, recusou tratamento posterior.

Tabela 2. Estatísticas descritivas em relação ao índice de astrócitos gemistocíticos e os índices da imuno-expressão positiva da p53, segundo o grau histológico.

\begin{tabular}{lccccccc}
\hline $\begin{array}{l}\text { Grau } \\
\text { Histológico }\end{array}$ & & Média & Mediana & $\begin{array}{c}\text { Desvio } \\
\text { padrão }\end{array}$ & $\begin{array}{c}\text { Erro } \\
\text { padrão }\end{array}$ & Mínimo & Máximo \\
\hline II $(\mathrm{n}=17)$ & IAG & 17,58 & 16,83 & 6,21 & 1,51 & 8,07 & 26,70 \\
& IAp53+ & 4,81 & 4,73 & 5,78 & 1,40 & 0,00 & 17,20 \\
& IAGp53+ & 0,27 & 0,00 & 0,71 & 0,17 & 0,00 & 2,75 \\
& III $(n=5)$ & 17,39 & 16,45 & 5,33 & 2,38 & 10,97 & 23,69 \\
& IAp53+ & 3,75 & 4,17 & 3,99 & 1,79 & 0,00 & 9,57 \\
& IAGp53+ & 0,12 & 0,00 & 0,16 & 0,07 & 0,00 & 0,30 \\
\hline
\end{tabular}

IAG, Índice de astrócitos gemistocíticos; IAp53+, Índice de astrócitos com imuno-expressão positiva da p53; IAGp53+, Índice de astrócitos gemistocíticos com imuno-expressão positiva da p53. 


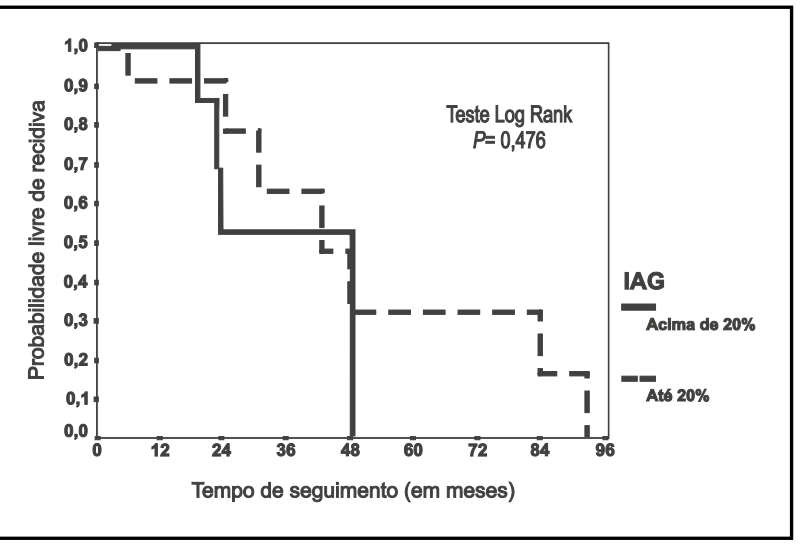

Fig 2. Curva de Kaplan Meier, de acordo com índice de astrócitos gemisticíticos (IAG).

inferior a $60 \%$. O tempo médio de recorrência estimado por este método foi de 45,3 meses (I.C. (95\%) $=[29,4 ; 61,2])$ (Tabela 1). Os 22 pacientes foram classificados em dois grupos: com IAG até $20 \%$ e acima de $20 \%$, e foi avaliado o tempo de recorrência através da curva de Kaplan Meier (Fig 2). Não há diferença estatisticamente significante, na probabilidade livre de recorrência, entre os dois grupos $(p=0,476)$. Como considerou-se qualquer percentagem de núcleos corados como imuno-expressão positiva para a p53, os pacientes foram divididos em dois grupos: com imuno-expressão positiva ou negativa para a p53. A curva de Kaplan Meier mostrou que não há diferença estatisticamente significante $(p=0,560)$, no intervalo até a recorrência, entre os dois grupos (Fig 3).

\section{DISCUSSÃO}

Astrocitomas gemistociticos são uma variante dos astrocitomas difusos caracterizada histologicamente pela presença predominante de astrócitos gemistocíticos. Entretanto vários autores autores ${ }^{13-15}$ não definem a quantidade mínima dessas células necessária para essa classificação. Krouwer et al. ${ }^{16}$ propuseram $20 \%$ como o valor mínimo para tal definição. Watanabe et al. ${ }^{11}$ em estudo de 28 astrocitomas gemistocíticos encontraram índice médio de gemistocitos de 35,0\%, variando entre $15,2 \%$ e 58,0\%. Em publicação recente, Kleihues et al. ${ }^{4}$, ao caracterizarem astrocitoma gemistocítico, consideram que os gemistocitos deveriam corresponder a aproximadamente $20 \%$ de todas as células e referem-se ao valor de 35,0\%, determinado por Watanabe et al. ${ }^{11}$, como o valor médio da fração de astrócitos gemistocíticos presentes. Na série estudada por Kösel et al. ${ }^{17} \mathrm{o}$ índice de gemistocitos variou entre $16 \%$ e $86 \%$.

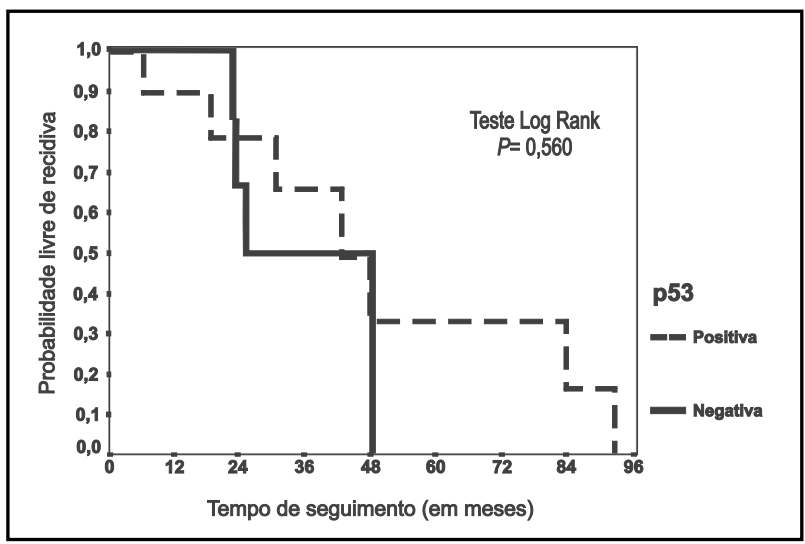

Fig 3. Curva de Kaplan M eier segundo a imuno-expressão da proteína p53.

Na presente casuística, o índice médio de astrócitos gemistocíticos foi de $17,53 \%$, com o menor valor encontrado $8,07 \%$ e o maior de $26,70 \%$. Nas neoplasias grau II OMS $(n=17)$ o índice médio foi de $17,58 \%$ e nas grau III $(n=5)$ foi de $17,39 \%$. O número de astrocitomas de baixo grau (grau II OMS) incluído neste estudo foi igual ao avaliado por Watanabe et al. ${ }^{11}$; entretanto, o índice médio de astrócitos gemistocíticos foi, aproximadamente, a metade do relatado por esses autores.

Embora os astrócitos gemistocíticos tenham sido descritos por Nissl em $1904^{18}$, que os interpretou como um subtipo de astrócitos encontrados em condições patológicas, quase um século depois seu verdadeiro papel ainda não foi completamente esclarecido ${ }^{1,2,12,18}$.

Com os dados obtidos, avaliou-se a relação entre índice de astrócitos gemistocíticos e recorrência da neoplasia. Neste estudo não foi observada relação, entre IAG até $20 \%$ ou superior e a recorrência da neoplasia $(P=0,476)$, pelo teste de Log Rank. A amostra pequena impossibilitou a aplicação de testes estatísticos na diferenciação de outros subgrupos, segundo o índice, maior ou menor, de gemistocitos. Watanabe et al. ${ }^{19}$ relataram 35 meses como o tempo médio para a progressão dos astrocitomas de baixo grau, contendo mais de $5 \%$ de gemistocitos, para astrocitoma anaplásico ou glioblastoma. Os astrocitomas de baixo grau, com menos de $5 \%$ de gemistocitos, apresentaram tempo médio significativamente maior, 64 meses. Mahler-Araújo ${ }^{20}$, avaliou a fração de gemistocitos em 60 pacientes com biópsias múltiplas. Observou diferença estatisticamente significante, no intervalo de tempo até a recurrência da neoplasia, entre astrocitomas de baixo grau, com mais de $5 \%$ de gemistocitos (média 
29,4 meses) e menor de 5\% (média 47,3 meses). Também nos casos com progressão, houve diferença estatisticamente significante no intervalo de tempo entre os casos com mais (média 29,5 meses) e com menos (média 51,1 meses) de 5\% de astrócitos gemistocíticos.

Superexpressão da proteína e mutação no gene p53 vêm sendo investigadas nos tumores astrocíticos, sendo correlacionadas com recorrência, acompanhada ou não por progressão para fenótipos mais malignos $^{6,9}$. Apenas pequeno número de trabalhos avaliou imuno-expressão da proteína p53 ou mutação do gene correspondente em astrocitomas, com especificação do subtipo gemistocítico ou com discriminação de gemistocitos ${ }^{9,11,17,21-24}$.

Neste estudo, a freqüência da imuno-reatividade da proteína p53 nuclear, considerando-se ambas as células neoplásicas, astrócitos e gemistocitos, foi de $54,54 \%$ (12/22), com índice médio de 4,57\%, sendo de $4,81 \%$ nas neoplasias grau II e de 3,75\% nas de grau III. Quando se considerou a imuno-expressão da p53 somente em astrócitos gemistocíticos, a positividade foi identificada em apenas sete dos casos (31,81\%), com índice médio de $0,24 \%$. Não se observou correlação entre o IAG e a imuno-expressão da proteína p53, nos astrocitomas, independentemente do grau histológico.

Em discordância com os resultados da presente série, os estudos de Yaziji et al. ${ }^{21}$, VITAL et al. ${ }^{23}$, Rathore et al. ${ }^{24}$ relatam frequência de $100 \%$ de positividade da p53 em astrocitomas gemistocíticos. Entretanto, as casuística são pequenas e há diferenças de ordem técnica. Cunningham et al. ${ }^{22}$ investigaram a imuno-expressão da proteína p53 em 120 gliomas, dos quais 20 (17\%) eram astrocitomas gemistocíticos. A frequência da positividade da p53 foi de $75 \%$ (15/20), com redução para 50\% (10/20), quando aplicado o valor de corte maior que $10 \%$.

Na investigação da imuno-reatividade da p53 em 40 astrocitomas de baixo grau (grau II OMS), com progressão histológicamente comprovada, Watanabe et al. ${ }^{19}$ encontraram índice médio de positividade da p53 em gemistocitos (7,4\%) significantemente maior $(P=0,0014)$ do que em ambas as células tumorais $(3,2 \%)$, sendo o índice em gemistocitos maior que $\mathrm{o}$ detectado neste estudo, $0,27 \%$.

Posteriormente, Watanabe et al. ${ }^{11}$ analisaram mutações no gene p53, através da técnica de PCR-SSPC, em 28 astrocitomas gemistocíticos, com índice médio de gemistocitos de 35,0\%. Identificaram mutações em $82 \%$ dos casos (23/28), todas localizadas no domínio central (exons 5-8).
Vários estudos tem mostrado correlações variáveis entre as técnicas moleculares e imuno-histoquímica, em tumores de diferentes histogenêses ${ }^{25-27}$.

Em consequência da diversidade dos padrões de mutações apresentada pelo gene p5328, a aplicação de um único método na investigação das mesmas pode ser insuficiente. Furuwatari et al. ${ }^{29}$ propõem a combinação de PCR-SSCP, FISH e imuno-histoquímica. Assim ao associarem esses métodos, os autores obtiveram $100 \%$ de eficiência.

Embora frequentes, mutações de ponto (missense) no gene p53, não são invariavelmente associadas a imuno-expressão da proteína p53. Mecanismos alternativos de estabilização da p53 têm sido relatados em aproximadamente $20 \%$ dos tumores ${ }^{30}$. Níveis detectáveis da p53 selvagem, podem ocorrer como resultado do incremento da transcrição e estabilização pós-transcricional, em resposta ao DNA danificado; outros mecanismos de inativação da p53 são exemplificados por sua interação com proteínas celulares e virais ${ }^{26}$.

Por outro lado, a negatividade da reação antígeno-anticorpo para detecção da p53, com presença de mutação no gene p53, pode ser atribuida a mutações frameshift ou nonsense, que produzem proteínas anômalas, com sítios antigênicos ausentes ou alterados, não sendo assim detectadas pelo método imuno-histoquímico ${ }^{26}$.

As diferenças, no espectro de mutações do gene p53, observadas em diferentes tumores e mesmo em um único tipo histológico, refletem a casualidade com a qual os acidentes genéticos ocorrem e se perpetuam através da seleção natural. Embora, sugira-se que o pior prognóstico dos pacientes com astrocitomas gemistocíticos possa estar relacionado a maior frequência de mutações no gene p53, tal interpretação deve ser feita com cautela, em consequência das múltiplas e complexas interações existentes na rota do gene p53, ainda não completamente conhecidas, assim como também não o éo papel dos gemistocitos.

\section{REFERÊNCIAS}

1. Hoshino T, Wilson CB, Ellis WG. Gemistocytic astrocytes in gliomas: an autoradiographic study. J Neuropathol Exp Neurol 1975; 34: 263281.

2. KrosJM, Stefanko SZ, DeJong AA, Van Vroonhoven CC, Van der Heul $\mathrm{RO}$, Van der Kwast TH. Ultrastructural and immunohistochemical segregation of gemistocytic subsets. Hum Pathol 1991; 22: 33-40.

3. Kros JM, Waarsenburg N, Hayes DP, Hop WCJ, Van Dekken H. Cytogenetic analysis of gemistocytic cells in gliomas. J Neuropathol Exp Neurol 2000;59:679-686.

4. Kleihues P, Davis RL, Ohgaki H, Burger PC, Westphal MM, Cavene WK. Diffuse astrocytomas. In Kleihues P, CaveneeWK (eds.). Pathology and genetics of tumours of the nervous system. Lyon: IARC, 2000: 22-26. 
5. Lang FF, Miller DC, Koslow M, Newcomb EW. Pathways leading to glioblastoma multiforme: a molecular analysis of genetic alterations in 65 astrocytic tumours. J Neurosurg 1994; 81: 427-436.

6. Watanabe K, Tachibana O, Sato K, Yonekawa Y, Klei hues P, Ohgaki H. Overexpression of the EGF receptor and p53 mutations are mutually exclusive in the evolution of primary and secondary glioblastomas. Brain Pathol 1996;6:217-224.

7. Kleihues $\mathrm{P}$, Ohgaki $\mathrm{H}$. Genetics of glioma progression and the definition of primary and secondary glioblastoma. Brain Pathol 1997;7:1131-1136.

8. Ohgaki H, Schaüble B, Zur Hansen A, Von Ammon K, Kleihues P. Genetic alterations associated with the evolution and progression of astrocytic brain tuours. Virchows Arch 1995;427:113-118.

9. Watanabe $K$, Sato $K$, Biernat $W$, et al. Incidence and timing of the $p 53$ mutations during astrocytoma progression in patients with multiple biopsies. Clin Cancer Res 1997; 3:523-530.

10. Kleihues $P$, CaveneeWK. Who classification of tumours of thenervous system. In: Kleihues P, Cavanee WK (eds). Pathology and genetics of tumours of the nervous system. Lyon: IARC, 2000: 6-7.

11. Watanabe K, Peraud A, Gratas C, Wakai S, Kleihues P, Ohgaki H. p53 and PTEN gene mutations in gemistocytic astrocytomas. Acta Neuropathol 1998;95:559-564.

12. Ohgaki $\mathrm{H}$, Watanabe $\mathrm{K}$, Peraud $\mathrm{A}$, et al. A case history of glioma progression. Acta Neuropathol 1999;97:525-532.

13. Burger PC, Scheithauer BW. Tumours of neuroglia and choroid plexus epithelium. In Rosai J (ed). Tumors of the central nervous system. Washington: Armed Forces Institute of Pathology, 1994: 25-106.

14. Ellison D, Love S, Chimelli L, et al. Astrocytic neoplasmas. In Ellison D, Love S (eds). Neuropathology. London: Mosby, 1998: 35.1-35.15.

15. McLendon RE, EnterlineDS, Tien RD, Thorstad WL, Bruner JM. Tumors of central neuroepithelial origin. In Bigner DD, McLendon RE, Bruner JM (eds.). Russell and Rubinstein's pathology of tumors of thenervous system. 6 Ed. London: Arnold Press, 1998: 307-571.

16. Krouwer HG], Davis RL, Silver P, Prados M. Gemistocytic astrocytomas: a reappraisal. J Neurosurg 1991, 74: 399-406.

17. Kösel S, Scheithauer BW, Graeber MB. Genotype-phenotype correlation in gemistocytic astrocytomas. Neurosurgery 2001;48:187-194.
18. Nissl, F. Zur histopathologie der paralytischen rindenerkrankung Histol Histopathol Arb Grosshirn 1904;1:316-494.

19. Watanabe K, Tachibana $\mathrm{O}$, Yonekawa Y, Kleihues P, Ohgaki, H. Role of gemistocytes in astrocytoma progression. Lab Invest 1997;76:277-284.

20. Mahler-A raújo MB. A nálise da participação de gemistocitos na recorrência e progressão das neoplasias astrocíticas difusas. Tese, Faculdade de Medicina de Ribeirão Preto. Ribeirão Preto, 2000.

21. Yaziji H, Massarani-Wajai R, Gujrati M, KuhnsJG, Martin AW, Parker $J C$. Role of p53immunohistochemistry in differentiating reactivegliosis from malignant astrocytic lesions. Am J Surg Pathol 1996; 20: 10861090.

22. Cunningham JM, Kimmel DW, Scheithauer RB, O'Fallon JR, Novotny PJ, Jenkins RB. Analysis of proliferation makers and p53 expression in gliomas of astrocytic origin: relationships and prognostic value. J Neurosurg 1997;86:121-130

23. Vital $\mathrm{A}$, Loiseau $\mathrm{H}$, Kantor $\mathrm{G}$, et al. $\mathrm{p} 53$ protein expression in grade II astrocytomas: immunohistochemical study of 100 cases with long-term follow-up. Pathol Res Pract 1998;194:831-836.

24. Rathore A, Kamarajan P, Mathur M, Sinha S, Sarkar C. Simultaneous alterations of retinoblastoma and $\mathrm{p} 53$ protein expression in astrocytic tumors. Pathol Oncol 1999;5:21-27.

25. Battifora H. p53immunohistochemistry: a word of caution. Hum Pathol 1994; 25: 435-436.

26. Bosari S, VialeG. The clinical significance of p53 aberrations in human tumours. Virchows Arch 1995; 427: 229-241.

27. Steilen-Gimbel H, Steudel W.I, Feiden W, Moringlane JR, Henn W, Zang KD. Genetic heterogeneity in human astrocytomas: spatial distribution of P16 and TP53 deletion in biopsies. Cancer Genet Cytogenet 1999; 113:115-119.

28. Hollstein M, Shomer B, Greenblatt M, Soussi T, Hovig E, Montesano R, Harris CC. Somatic point mutations in thep53 gene of human tumours and cell lines: updated compilation. Nucl Acids Res 1996;24:141-146.

29. Furuwatari $C$, Yagi A, Yamagami O, et al. A comprehensive system to explore p53 mutations. Am J Clin Pathol 1998;110:368-373.

30. Harris CC, Hollstein M. Clinical implications of the tumor suppressor gene. N Engl J Med 1993; 329:1318-1327. 